\title{
Image sensor market set for cyclic growth
}

\begin{abstract}
The market for image sensors will decline to $\$ 6.4$ billion in 2009 - a drop of $11 \%$ from 2008 - according to market analysts Strategies Unlimited. This is the first decline in the market since the company began tracking the industry in 1997. The image sensor market has enjoyed a compound annual growth rate of around $22 \%$ since 1997, and Strategies Unlimited predicts the average growth rate will return to single-digits in coming years.

"The current global economic climate is causing a temporary decline, but cycles will have a stronger effect in the future because the market forces that caused the rapid growth in image sensor revenues for cameraphones have matured," said Tom Hauskens, director of photonics at Strategies Unlimited. The company predicts that, over the next few years, there will still be an overall growth in unit sales for image sensors, but shortterm fluctuations in demand, periods of oversupply and shortage, and severe price pressure will make staying competitive more challenging.

One of the major changes in the market over the past few years has been in
\end{abstract}

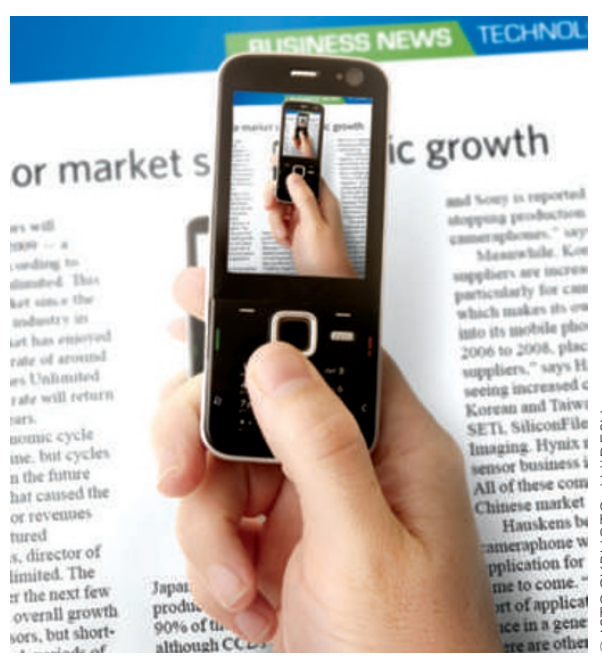

Asia. Japan once dominated world image sensor production, and still manufactures over $90 \%$ of the world's CCDs. However, although CCDs continue to dominate in digital cameras and security cameras, several Japanese companies are halting their production of image sensors. "Fujifilm stopped production of image sensors for its line of cameras in 2007, and Sony is reported to be thinking of stopping production of CMOS arrays for cameraphones," says Hauskens.

Meanwhile, Korean and Taiwanese suppliers are increasing their market share, particularly for cameraphones. "Samsung, which makes its own image sensors to go into its mobile phones, grew $61 \%$ from 2006 to 2008, placing it among the top suppliers," says Hauskens. "We are also seeing increased competition from other Korean and Taiwanese companies, such as SETi, SiliconFile Technologies, and PixArt Imaging. Hynix re-entered the image sensor business in the second half of 2008 . All of these companies are focusing on the Chinese market for handsets."

Hauskens believes that the cameraphone will remain the main application for image sensors for some time to come. "The cameraphone is the sort of application that comes along only once in a generation," he says. "Although there are other interesting applications such as medical devices, security cameras and automotive sensors, the cameraphone market is by far the biggest for image sensors today."

\section{Cognex wins patent dispute}

Cognex Corporation has accepted a settlement offer from Yxlon International $\mathrm{GmbH}$ and its US subsidiary in a dispute concerning the importation and sale of equipment containing its MVTec HALCON machine vision software. The settlement terms between the parties were not made public. Cognex alleged that HALCON software, marketed by MVTec Software $\mathrm{GmbH}$, infringes at least three Cognex machine vision patents. The International Trade Commission is investigating 20 companies for violating Cognex patents by selling, importing and using MVTec products that contain machine vision technology invented and patented by Cognex. A prior settlement with Multitest Electronische Systeme GmbH was announced earlier in the year.

"The recent successes of Cognex in this matter validates the strength of our patents and the claims that we have asserted," said Todd Keebaugh, vice president of legal affairs at Cognex. "Cognex is pleased with Yxlon's decision to seek settlement of this matter, and we value this as an opportunity to move forward and build a successful partnership with Yxlon and its worldwide affiliates."

\section{Automotive imaging \\ deals announced}

Two imaging companies have recently announced deals in the automotive industry that involve sophisticated imaging technology relating to 360 -degree vision. ImmerVision has signed a licensing agreement with Samsung ElectroMechanics to produce and commercialize 'panomorph' optics for the automotive market, and OmniVision Technologies' AutoVision image sensors have been selected by Altek Corporation for its 'Eagle View' 360-degree surround viewer and lane departure warning system (LDWS).

Imaging applications relating to driver assistance are becoming increasingly popular in today's vehicles. According to market research firm Techno Systems Research, the percentage of integrated vehicle cameras featuring CMOS image sensors is projected to increase from around $20 \%$ in 2008 to more than $70 \%$ in 2013 .

Altek's camera-based LDWS utilizes OmniVision's CMOS image sensors, and are mounted behind the windshield or rear view mirror of the vehicle to continuously track visible lane markings. The control unit monitors the roadway traffic control lines with image recognition software to calculate the lateral and angular divergence from the lane's centre. The LDWS then estimates the vehicle's future position through sophisticated algorithms. If the driver fails to use the indicator and the data suggests the vehicle is leaving its intended path, the system alerts the driver through an audio and visual warning on the vehicle's instrument panel.

Immervision's technology is based on a unique 'panomorph' lens technology that enables a 360-degree image to be captured from a standard camera, without distortion.

"Samsung Electro-Mechanics, known for its global leadership in vision sensors for the mobile phone industry, is now taking a leadership position in the automotive industry by embracing new immersive panoramic solutions that use ImmerVision's advanced panomorph technology. This will make next-generation cars more secure, and the driver's journey more enjoyable," said Alain Marchildon, vice president of custom panoramic applications at ImmerVision. 